\title{
La creación de valor a través de la planeación estratégica en microempresas emprendedoras
}

Creation of value through strategic planning in microenterprises

\author{
Jorge Armando López Lemus ${ }^{1 *}$ María Teresa De la Garza Carranza \\ ${ }^{1}$ Universidad de Guanajuato, México \\ ${ }^{2}$ Instituto Tecnológico de Celaya, México
}

Recibido el 28 de noviembre de 2018; aceptado el 2 de julio de 2019

Disponible en Internet el: 29 de julio de 2019

\section{Resumen}

Propósito: El propósito de la investigación es identificar la influencia que existe de la planeación estratégica sobre la creación de valor en las microempresas emprendedoras de la región del bajío del Estado de Guanajuato, México.

Diseño y Metodología: El diseño metodológico fue cuantitativo, explicativo, observacional y transversal. Se empleó una muestra de 407 jóvenes emprendedores. Se desarrolló un modelo de ecuación estructural (SEM). En cuanto a los índices de bondad y ajuste del SEM $\left(\mathrm{X}_{2}=146.10 \mathrm{gl}=52 ; \mathrm{CFI}=0.969\right.$ y TLI=0.960; RMSEA=.06) fueron absolutamente aceptables.

Resultados: Se demuestra que existe relación positiva y significativa entre la planeación estratégica y la creación de valor en el emprendimiento de las microempresas. Asimismo, la planeación estratégica influye positiva y significativamente sobre la creación de valor en el emprendimiento de las microempresas Originalidad / Valor: Los hallazgos son relevantes y de gran valor ya que actualmente no hay suficientes investigaciones que estén enfocados en las variables analizadas.

\footnotetext{
*Autor para correspondencia

Correo electrónico jorge.lemux@hotmail.com (J.A .López Lemus).

La revisión por pares es responsabilidad de la Universidad Nacional Autónoma de México.

http://dx.doi.org/10.22201/fca.24488410e.2020.2312

0186- 1042/@ 2019 Universidad Nacional Autónoma de México, Facultad de Contaduría y Administración. Este es un artículo Open Access bajo la licencia CC BY-NC-SA (https://creativecommons.org/licenses/by-nc-sa/4.0/)
} 
Código JEL: M10, M13

Palabras Clave: Planeación estratégica; Creación de valor; Emprendimiento

\begin{abstract}
Purpose: The purpose of the research is to identify the influence that exists of strategic planning on the creation of value in the entrepreneurial microenterprises of the Bajio region of the State of Guanajuato, Mexico.

Design and Methodology: The methodological design was quantitative, explanatory, observational and transversal. A sample of 407 young entrepreneurs was employed. A structural equation model (SEM) was developed. As for the SEM goodness and adjustment indices (X2=146.10 gl=52; CFI=0.969 y TLI=0.960; RMSEA=.06) they were acceptable.

Results: It is demonstrated that there is a positive and significant relationship between strategic planning and the creation of value in the entrepreneurship of micro-enterprises. Likewise, strategic planning has a positive and significant influence on the creation of value in the entrepreneurship of micro-enterprises. Originality / Value: The findings are relevant and of great value since currently there are not enough researches that are focused on the analyzed variables
\end{abstract}

JEL Code: M10, M13

Keywords: Strategic planning; Value creation; Entrepreneurship

\title{
Introducción
}

El emprendimiento es una alternativa a los bajos salarios que ofrecen las empresas a la nueva generación de los "millennials" o generación Y que recién inicia su carrera profesional. Esta generación de trabajadores se caracteriza por presentar diferencias importantes de otras, como la generación X (nacidos entre 1965 a 1980) o los "Baby Boomers" (nacidos entre 1946 a 1964). Los trabajadores Y manejan de forma efectiva las redes sociales, buscan estar cerca de su familia y amigos y crecieron con valores de ética con referencia al medio ambiente (Dries, Pepermans \& De Kerpel, 2008, Lissitsa \& Kol, 2016, Nitya \& Anand, 2016). Los trabajadores del milenio buscan áreas de oportunidad para generar riqueza, y dependencia económica (López-Lemus, De la Garza, 2019) y con ello, contribuir al desarrollo económico de la región.

De acuerdo con López (2018) en México el 75\% de los emprendimientos fracasan. En este mismo sentido, el Instituto Nacional del Emprendedor (INADEM) y el Instituto del Fracaso en México ha señalado que el fracaso de los emprendimientos es multifactorial (López-de-Alba, 
Zavala, De la Garza, López-Lemus, \& Ramos, 2016). Algunas razones de este fracaso son un el plan de negocio mal estructurado, falta de planes estratégicos, la poca creación de valor, así como el pobre rendimiento financiero (Kirkbesoglu \& Ozder, 2015; Owen et al., 2019).

Ante la problemática señalada anteriormente, es menester que los emprendedores de las microempresas necesitan impulsar, desarrollar y aplicar una planeación estratégica que les permitan identificar sus fortalezas y debilidades (Arend et al., 2017; Teixeira \& Canciglieri, 2019), así como también identificar áreas de oportunidad y valorar las amenazas con la finalidad de generar estrategias tácticas y operativas (Jaafreh \& Al-abedallat, 2013) que contribuyan a mantenerse en un mercado competitivo y ser resilientes ante nuevos escenarios económicos. Es por ello que, la creación de valor a través de los productos y servicios son algunos de los factores importantes que mantendrá a la microempresa en el mercado, pues este factor podría representar componentes diferenciadores ante su competencia.

Generalmente la formulación de una estrategia responde a los cambios que el propio emprendedor ve en el entorno. De tal manera que generalmente la creación de valor va a depender de las habilidades cognitivas y perceptuales del administrador de la organización (Parnell, Lester \& Maneffee, 2000). A diferencia de las grandes empresas, las empresas pequeñas se caracterizan por la falta de recursos, debido a esto, es difícil que los encargados de las empresas realicen una planeación formal. Por esta razón, generalmente hacen diferenciaciones en costos (Wolff \& Pett, 2000). Por lo tanto, la planeación estratégica es considerada como uno de los factores que tiene una fuerte relación con la creación de valor (Oliva \& Kotabe, 2019), por lo que ambos constructos juegan un papel fundamental en lograr un rendimiento empresarial de la microempresa (De la Garza, Zavala \& López-Lemus, 2017), y con ello, posicionarse en el mercado ante un ambiente competitivo (Ansoff y McDonnell, 1990; Glaister y Falshaw, 1999; Kaplan \& Beinhocker, 2003; Spender et al., 2017; Teberga et al., 2018).

El objetivo principal de la presente investigación es conocer y analizar la influencia que ejerce la planeación estratégica sobre la creación de valor en las microempresas de la región del bajío del estado de Guanajuato, México. De manera específica, se pretende conocer y analizar la relación que existe entre la planeación estratégica y la creación de valor de las iniciativas empresariales del bajío, de tal manera que se permita establecer recomendaciones para la implementación de cursos a los futuros emprendedores en los diversos centros de emprendimiento. 


\section{Marco Teórico}

\section{Planeación Estratégica}

Uno de los principales rasgos de los jóvenes pertenecientes de la generación Y o Millennial es la individualidad que muestran al desarrollo de sus actividades o tareas, así como también, la búsqueda de reivindicar su autonomía a través de sus acciones, formas de pensar y de llevar acabo proyectos personales. Ante la autonomía que les caracteriza, esta generación refleja una tendencia de emprender e iniciar nuevas empresas (González, 2011). Esta iniciativa que muestran a través de su comportamiento, tiende a generar modelos de negocio sin considerar los riesgos (Ghezzi, 2014) que se puede tener al emprender un modelo de negocio. Wheadon \& Duval-Couetil (2017), encontraron que debido a que existe una mejor formación de los jóvenes en relación con el emprendimiento (Frunzaru \& Cismaru, 2018) están más preparados para crear y desarrollar empresas. De tal manera, si el joven emprendedor emplea habilidades de gestión tales como llevar a cabo una planeación estratégica aunado con su iniciativa y autonomía tendrá una visión más clara sobre las estrategias tácticas y operativas para lograr el éxito a través del posicionamiento de la microempresa en el mercado. La planeación estratégica es una herramienta que permite a los líderes de diferentes tipos de empresas y organizaciones alcanzar los objetivos empresariales a través de estrategias tácticas y operativas a corto plazo, mediano y a largo plazo (Kaplan \& Beinhocker, 2003; Peng, 2012). Debido a la relevancia y factor de éxito que se ha tenido actualmente está herramienta, la planeación estratégica es utilizada en el ámbito empresarial.

A falta de conocimiento sobre las prácticas de gestión empresarial, los Millennial gustan de tomar decisiones sin evaluar los beneficios o perjuicios que estas contraen debido a que no tienen una visión clara sobre los objetivos a alcanzar (Lissitsa \& Kol, 2016; Nitya \& Anand, 2016), Es por ello que, los investigadores consideran que la planeación estratégica es un constructo que facilita la implementación y la alineación de las estrategias tácticas y operativas para lograr los objetivos a través de la toma de decisiones (Kohtamäki et al., 2012). En este sentido, la planeación estratégica es definida como un proceso estructural en la que se establecen los objetivos a largo y a corto plazo de la organización, así como las estrategias que se deberán implementar para cada una de las metas establecidas a largo plazo a través de indicadores clave (KPI's: Key performance indicator) por medio de un control de mando integral (Kahla, 2017) con la finalidad de lograr la visión empresarial a través de alcanzar los objetivos establecidos y con ello, ser más competitivo (Peng, 2012) y posicionarse en el mercado. 
La planeación estratégica representa una de las herramientas para la toma de decisiones (Vecchiato, 2019) y la más utilizadas en el emprendimiento a través de las prácticas de gestión administrativa empresarial (Posch \& Garaus, 2019). Considerando que, una de las principales funciones de la planeación estratégica radica en orientar, acelerar y facilitar la integración de la sostenibilidad en el proceso y desarrollo del producto o servicio con valor agregado mediante cambios en la gestión empresarial a través de la estrategia (Arend et al., 2017; Teixeira \& Canciglieri, 2019). Por lo que entonces, el desarrollo y aplicación de la planeación estratégica dentro del sector empresarial es empleada como una ventaja competitiva que favorece en la permanencia en un mercado altamente competitivo.

La alta dirección, así como los líderes emprendedores son los encargados de establecer los objetivos y las estrategias que permitirán alcanzar a corto y a largo plazo cada una de las metas establecidas, a través de la planeación estratégica (Krumwiede \& Charles, 2006). De acuerdo con los estudios realizados por Khan y Al-Buraki (1992), el grado de utilización de las principales herramientas de estrategia más empleadas por los líderes emprendedores, directivos ejecutivos y gerentes de organizaciones empresariales a través de sus prácticas de gestión empresarial son: (1) el análisis financiero (62\%), (2) el análisis FODA (55\%), (3) el análisis de brechas (21\%) y finalmente, (4) el análisis de espacio (21\%). Estas herramientas son necesarias para mejorar las prácticas de gestión empresarial de los jóvenes emprendedores, y la aplicación de estas, contribuirá en garantizar estratégicamente el éxito de la microempresa emprendedora.

Debido a la relevancia que tiene el uso y aplicación de la planeación estratégica dentro del sector empresarial, representa una de las prácticas de la gestión y dirección administrativa de la empresa (Jaafreh \& Al-abedallat, 2013; Ooi, 2014; Shammari \& Hussein,2008) que contribuye en el método de gestión de calidad de Deming a través las prácticas de gestión de una empresa (Deming, 1986). Así mismo, es uno de los constructos que conforma la gestión de la calidad total (TQM: Total Quality Management), por lo que es medida a través del instrumento TQM (Lee et al., 2012, Prajogo \& Sohal, 2003) desarrollado por Ahire, Golhar y Waller (1996).

Por tanto, la planeación estratégica representa una de las estrategias que contribuye en la generación y desarrollo de nuevos productos y servicios, así como establecer y aplicar planes estratégicos enfocados en el posicionamiento tanto del producto como del servicio a través de la creación de valor, representando este último, como una ventaja competitiva y diferenciadora idealizada estratégicamente ante su competencia. Esto es especialmente importante para empresas que comienzan su desarrollo (von Gelderen, Frese \& Thurik, 2000) 


\section{Creación de Valor}

Una de las principales características de los Millennial radica en la habilidad creativa que cuentan para desarrollar y encontrar nuevas formas de darle utilidad a los objetos (Lissitsa $\&$ Kol, 2016). Esta habilidad les permite encontrar diferentes usos a las cosas, pero también, promueven a realizar ciertos cambios a los productos y servicios para que estos sean más novedosos e innovadores, de mayor calidad, bajo en costo y de pronta respuesta. En este sentido, los Millennial tienen un potencial en crear valor a nuevos productos o servicios para satisfacer necesidades que han identificado anteriormente (Pillai \& Dam, 2019) con finalidad de obtener un beneficio económico.

La creación de valor es definida como la capacidad que tienen las empresas para generar utilidades a través de una actividad económica (Porter \& Kramer, 2006). Para Viscarri (2011) es ofrecer algo a una persona que desea satisfacer alguna necesidad retribuyendo con algo, que generalmente es económico.

De acuerdo con Sánchez y Cerdán (2002), actualmente las empresas enfrentan un nuevo escenario competitivo, donde la creación de valor empresarial surge a partir de la capitalización de los activos intangibles que ofrece la empresa. En este sentido, se hace referencia al servicio, la flexibilidad en entregas de productos, correcciones en los volúmenes de producción, la calidad en el desarrollo de productos y la prestación de servicios (Cousens et al., 2009; De Toni \& Tonchia, 1998), entrega a tiempo, y la calidad ofrecida a través de los productos y servicios (Prajogo \& McDermott, 2008; Coelho \& Augusto, 2010).

Ante estas formas de capitalización empresarial, los Millennial tienen un fuerte potencial y habilidad de generar valor a los productos y servicios a través de la identificación de los intangibles que satisfacen las necesidades de un sector de mercado. Por lo que, esta habilidad se convierte en una ventaja competitiva que le permitirá fácilmente introducirse y posicionarse en el mercado, logrando sostenibilidad y sustentabilidad tanto económica como financiera por medio del rendimiento de la microempresa emprendedora (Kim, 2018).

Para la creación de valor, Noordin et al. (2015), considera que el rendimiento financiero como el no financiero son factores que contribuyen a este factor en las empresas a través de los resultados del rendimiento empresarial. En este sentido, el rendimiento financiero hace referencia a la utilidad generada a través de la inversión (ROI) y la rentabilidad obtenida a través de los recursos propios de la empresa (ROE). Por otro lado, el rendimiento no financiero es definida a partir de la creación de valor por medio de la capitalización de la empresa considerando los activos intangibles (Perrini \& Vurro, 2010) como el servicio y flexibilidad que se ofrece a través de los clientes, en términos de calidad, entrega a tiempo, costos, volumen 
de venta, el grado de innovación de los productos y servicios que ofrece la empresa (Noordin et. Al, 2015; Verdú-Jover et al., 2004).

Grönroos y Voima, (2013), considera que existen tres etapas importantes en el proceso de creación de valor (1) la esfera del proveedor: el proveedor desarrolla y proporciona recursos para potencializar y respaldar la creación de valor de un cliente, (2) la esfera del cliente: el cliente potencializa la creación de valor a través de sus necesidades y (3) la esfera conjunta: el proveedor y el cliente interactúan en forma indirecta en la creación de valor (Brozovic, Nordin \& Kindström, 2016).

Asimismo, Teixeira y Canciglieri (2018) consideran que el desarrollo y la aplicación de la planeación estratégica es una de las principales prácticas de gestión empresarial de cualquier sector y tamaño de la empresa (López-Lemus \& De la Garza, 2019) que debe considerar el gestor o el emprendedor, debido a que promueve la generación de valor a través del proceso sostenible de desarrollo del producto o servicio que este generando la microempresa. Ante la envergadura que tiene la planeación estratégica en relación con la creación de valor, el sector empresarial la emplea como una de las principales herramientas que contribuyen a mejorar el modelo de negocio a través de generar valor tanto como al producto como al servicio. En este sentido, la planeación estratégica promueve la creación de valor y ambas representan una ventaja competitiva (Teixeira \& Canciglieri, 2019) que permitirá generar un rendimiento económico de la microempresa emprendedora. Por lo tanto, la planeación estratégica y su relación con la creación de valor juegan un papel importante para lograr el éxito (Wolf \& Floyd, 2017) de las microempresas emprendedoras.

Para la presente investigación se consideró la creación de valor a partir del rendimiento no financiero generado a partir de los activos intangibles que ofrece la microempresa hacia sus clientes. Por lo que, los jóvenes emprendedores necesitan desarrollar una planeación estratégica donde puedan generar acciones inteligentes para producir u ofrecer servicios por medio de la diferenciación con su competencia en el mercado a partir del rendimiento no financiero a través de los intangibles que ofrece la microempresa emprendedora. En este sentido, el objetivo principal de la presente investigación es conocer la influencia que existe de la planeación estratégica sobre la creación de valor en las microempresas de la región del bajío del estado de Guanajuato, México; y como objetivo particular radica en, conocer la relación que existe entre la planeación estratégica y la creación de valor en la iniciativa de microempresas de la región del bajío del estado de Guanajuato, México.

A partir de los objetivos planteados, se establecen las siguientes hipótesis:

$\mathrm{H}_{1}$ : La planeación estratégica influye positiva y significativamente sobre la creación de valor en la iniciativa de microempresas de la región del bajío del estado de Guanajuato, México. 
$\mathrm{H}_{2}$ : Existe una correlación positiva y significativa entre la planeación estratégica y la creación de valor en la iniciativa de microempresas de la región del bajío del estado de Guanajuato. México.

Para evaluar las hipótesis señaladas anteriormente, se diseñó un modelo de ecuaciones estructurales (SEM) hipotético, ver figura 2.

\section{Metodología}

La presente investigación es un estudio cuantitativo, explicativo y correlacional, debido a las características de medición que presentan las variables elegidas, mismas que pretenden, dar una visión general y aproximada a una determinada realidad (Hernández-Sampieri \& Mendoza, 2018), en este sentido, la influencia que ejerce la planeación estratégica sobre la creación de valor en las microempresas de la región del bajío del estado de Guanajuato, México. El tipo de estudio fue transversal, en razón al periodo y secuencia del estudio, para ello se aplicaron instrumentos en una sola ocasión, en un mismo momento a los sujetos del estudio, es decir, se realizó un corte en el tiempo, con la finalidad de obtener la información necesaria para el análisis y medición de las variables (Hernández-Sampieri \& Mendoza, 2018).

\section{Muestra}

La población estudiada a través de esta investigación son parte de la generación Millenians, mismos que, se encuentran emprendiendo nuevos modelos de negocio realizando funciones de administración y de gestión empresarial (Gursoy et al., 2013; Kupperschmidt, 2000), debido a sus características de personalidad (Parment, 2013) y su influencia del uso y manejo de la tecnología (Gurau, 2012; Lissitsa \& Kol, 2016; Prior, Hubbard \& Rai, 2016).

El tipo de muestreo fue no probabilístico intencional, debido a que se requería obtener el mayor número de microempresas participantes. En el marco de muestreo empleado en la presente investigación, fueron sujetos de investigación jóvenes emprendedores, líderes de proyectos de micro organizaciones empresariales industriales, comercio y de servicio de la región del bajío del estado de Guanajuato, México (Ver figura 1). Los criterios de inclusión fueron jóvenes emprendedores de microempresas que contaran al menos de un año de experiencia en el emprendimiento de un negocio, producto o servicio. Para recolección de los datos, se diseñó una plataforma alojado a un servidor de internet donde los participantes ingresaron para contestar los instrumentos empleados para su análisis cuantitativo. De tal manera, que se 
aplicaron los instrumentos a los 407 jóvenes emprendedores de microempresas en el periodo correspondiente al día 9 de Julio del 2018 al 13 de Abril del 2019.

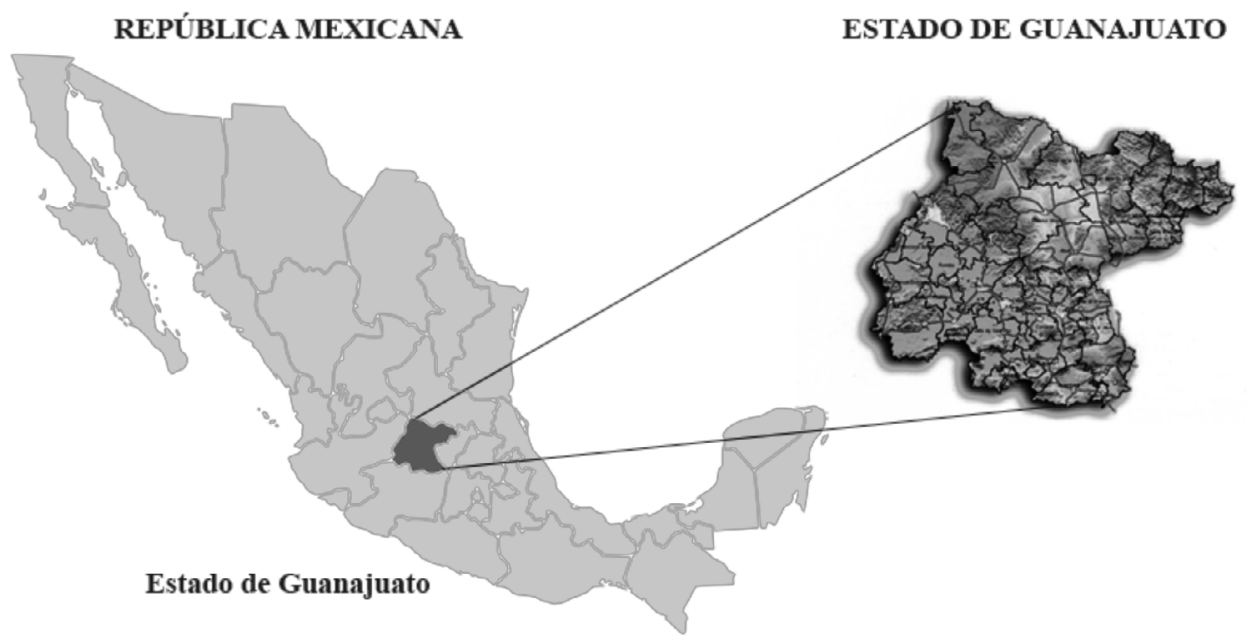

Figura 1. Ubicación espacial de la región del bajío estado de Guanajuato dentro de la República Mexicana. Basado en López-Lemus (2019).

Nota: En la figura se hace referencia a la ubicación del estado de Guanajuato en la República Mexicana y donde se realizó la recolección de la información de los jóvenes emprendedores de microempresas guanajuatenses.

De los cuales, el $44.2 \%(n=180)$ fueron hombres mientras que el $55.8 \%(n=227)$ fueron mujeres. Con respecto a la edad de los participantes, el 33.4\% $(n=136)$ tiene una edad de menor a 20 años, el $45.7 \%$ ( $n=186)$ cuenta con una edad de 21 a 25 años, el $12.8 \%(n=52)$ tiene una edad de 26 a 30 años, el 3.4\% ( $n=14)$ cuenta con una edad de 31 a 35 años y finalmente, el $4.7 \%(\mathrm{n}=19)$ tiene una edad de más de 35 años. En cuanto al grado de estudios de los sujetos, el 30.2\% ( $n=123$ ) cuenta con un nivel de estudios de bachillerato, el 64.9\% ( $n=264)$, cuenta con un grado de estudios de nivel de licenciatura, mientras que, el $4.9 \%(n=20)$ tiene un nivel de estudios de grado de posgrado. En cuanto al giro de las microempresas, el 26.3\% ( $\mathrm{n}=107)$ fueron de del sector comercial, el 40.5\% ( $\mathrm{n}=165)$ representaron empresas del sector industrial y finalmente, el 33.2\% ( $n=135)$ fueron del sector de servicios.

Para la selección de las microempresas, se consideró la estratificación del tamaño de empresas en México, de acuerdo con el Diario Oficial de la Federación (DOF) (2009) de México, se consideró la estratificación de acuerdo con el número de empleados, es decir, se consideraron las empresas compuestas por 1 hasta 10 empleados. 
Para el análisis de los datos obtenidos, se empleó el software estadístico SPSS Statistics v.21 y para la comprobación de las hipótesis establecidas, se desarrolló un modelo de ecuación estructural (SEM), a través del software estadístico Amos v. 21.

\section{Confiabilidad y Validez de Instrumentos}

De acuerdo con los análisis de datos de las variables observadas de los constructos, no siguieron una distribución normal. Sin embargo, Bollen y Stine (1992) y Hair et al. (2017) sostienen que las técnicas de bootstrapping representan un medio para corregir, por un lado, problemas en situaciones del tamaño de muestra y por otro, que los datos no sigan una distribución normal. Asimismo, las técnicas de bootstrapping proporciona un valor de significancia ( $p$ ) sin asumir la normalidad de la muestra y corrige el valor probabilístico proporcionado por el método de máxima verosimilitud para contrastar el ajuste global (Davison \& Hinkley1997; Efron \& Tibshirani, 1993; Hair et al., 2017).

Para la validez de los instrumentos utilizados, se consideró la validez de constructo debido a que es el principal de los diferentes tipos de validez. En este sentido, "la validez de constructo es el concepto unificador que integra las consideraciones de validez de contenido y de criterio en un marco común para probar hipótesis acerca de relaciones teóricamente relevantes" (Messick, 1980; p.1015). Asimismo, Cronbach (1984) considera que "la meta final de la validación es la explicación y comprensión y, por tanto, esto nos lleva a considerar que toda validación es validación de constructo" (p.126).

2.2.1 Planeación estratégica. Para medir este constructo de los jóvenes líderes emprendedores, se empleó la dimensión de planeación estratégica del instrumento de prácticas de gestión empresarial TQM (ver anexo) desarrollado por Ahire, Golhar y Waller (1996). Este instrumento está conformado por 7 ítems. Los reactivos tienen un formato tipo Likert con 5 puntos de repuesta, donde 1 representa "Totalmente en desacuerdo" y 5 "Totalmente de acuerdo". Para evaluar la confiabilidad de este instrumento se empleó el Alfa de Cronbach $(\alpha=0.908)$, misma que resultó satisfactorio (Cronbach, 1951; Hair et al., 2017).

En cuanto a la validez de constructo, se desarrolló un análisis factorial confirmatorio (CFA) basado en Ahire, Golhar y Waller (1996) a través de un modelo de ecuación estructural bajo la técnica de Bootstrapping y el método de máxima verosimilitud (ML) por medio de un remuestreo de 1,000 Bootstraps. Para la validación del SEM, se consideró la prueba de Chi-cuadrado $\left(X^{2}=41.23 / g l=13\right)$, el índice comparativo de ajuste (CFI=0.982), el índice de Tucker-Lewis (TLI=0.972), Índice de la Aproximación de la Raíz Cuadrada del Error Cuadrático Medio (RMSEA=0.07), por lo que los índices de bondad y ajuste del modelo 
resultaron ser absolutamente satisfactorios (Asparouhov, Hamaker \& Muthen, 2018; Bollen, 1989; Jöreskog \& Sörbom, 1981; Muthén \& Muthén, 1998-2007; Rigdon, 1996). Asimismo, se comprobó la validez convergente a través de las cargas factoriales estandarizadas $(\lambda)$ de las variables observables mismas que fueron superiores a .40 (Hair et al., 2017; Jöreskog \& Sörbom, 1981; Muthén, 2001; Muthén 2002;) con un alto nivel de significancia. Ver Tabla 2.

2.2.2 Creación de Valor. Para medir este constructo, se empleó la dimensión del rendimiento no financiero del instrumento resultados del rendimiento (ver anexo) adaptado por Noordin et al. (2015). El instrumento está conformado por 5 ítems. Los reactivos tienen un formato tipo Likert con 7 puntos de repuesta, donde 1 representa "pobre rendimiento" y 7 "excelente rendimiento". Para evaluar la confiabilidad de este instrumento se empleó el Alfa de Cronbach $(\alpha=0.881$ ), misma que resultó satisfactorio (Cronbach, 1951; Hair et al., 2017).

En cuanto a la validez del constructo, se desarrolló un análisis factorial confirmatorio (CFA) a través de un modelo de ecuación estructural bajo la técnica de Bootstrapping y el método de máxima verosimilitud (ML) por medio de un remuestreo de 1,000 Bootstraps. Para la validación del SEM, se consideró la prueba de Chi-cuadrado $\left(X^{2}=7.80\right.$ / gl=4), así como también, los índices de bondad y ajuste (CFI=0.996; TLI=0.991; RMSEA=0.04), por lo que los índices del modelo resultaron ser absolutamente satisfactorios (Asparouhov, Hamaker \& Muthen, 2018; Bollen, 1989; Jöreskog \& Sörbom, 1981; Muthén \& Muthén, 1998-2007; Rigdon, 1996). Asimismo, se comprobó la validez convergente a través de las cargas factoriales estandarizadas $(\lambda)$ de las variables observables mismas que resultaron satisfactorias (Hair et al., 2017; Jöreskog \& Sörbom, 1981), ver tabla 3.

Tabla 1

Estadística descriptiva: Promedios, desviación estándar, varianza, y correlación de las variables

\begin{tabular}{llllll}
\hline VARIABLES & Media & D.S. & Varianza & 1 & 2 \\
\hline Planeación Estratégica & 3.80 & 0.82 & 0.68 & 1.000 & \\
Creación de Valor & 5.17 & 1.19 & 1.43 & $0.686^{* *}$ & 1.000 \\
\hline$(* * \mathrm{p}<0.001)$ & & & &
\end{tabular}

$(* * \mathrm{p}<0.001)$
Fuente: Diseño Propio

Nota: En la tabla se muestran los estadísticos descriptivos, así como la correlación entre las variables planeación estratégica y creación de valor. En este sentido, se puede apreciar que la correlación entre las variables $(r=0.686$; $p<0.001)$ es positiva y significativa. 


\section{Tabla 2}

Cargas factoriales estandarizadas y alfa de Cronbach la planeación Estratégica

\begin{tabular}{lc}
\hline Ítems & Carga Factorial $(\lambda)$ \\
\hline $\begin{array}{l}\text { PE1.- Nuestra organización tiene una misión que se ha comunicado a través de la com- } \\
\text { pañía y es apoyada por nuestros empleados. }\end{array}$ & $0.760^{* *}$ \\
$\begin{array}{l}\text { PE2.- Nuestra organización tiene un proceso de planificación integral y estructurado que } \\
\text { establece y regula regularmente las metas a corto y a largo plazo. }\end{array}$ & $0.821^{* *}$ \\
$\begin{array}{l}\text { PE3.-Nuestra organización siempre incorpora las capacidades de los proveedores y las } \\
\text { necesidades de otras partes interesadas, incluida la comunidad, cuando desarrollamos } \\
\text { nuestros planes, políticas y objetivos. }\end{array}$ & $0.766^{* *}$ \\
$\begin{array}{l}\text { PE4.- Nuestra organización tiene una declaración por escrito una estrategia que abarca } \\
\text { las operaciones de negocio que este articulado y acordado por nuestro gerente superior. }\end{array}$ & $0.770^{* *}$ \\
$\begin{array}{l}\text { PE5.- Los planes estratégicos (y los planes tácticos) están vinculados a los valores de } \\
\text { calidad. }\end{array}$ & $0.811^{* *}$ \\
$\begin{array}{l}\text { PE6.- Se hace inclusión de mejoras continuas de la calidad en el proceso de planifica- } \\
\text { ción. }\end{array}$ & $0.782^{* *}$ \\
$\begin{array}{l}\text { PE7.- Las quejas de los clientes fueron analizadas y utilizadas para la mejora del produc- } \\
\text { to / servicio que ofrece la organización. } \\
\text { TLI }\end{array}$ & $0.658^{* *}$ \\
$\begin{array}{l}\text { CFI } \\
0.982\end{array}$ & $\begin{array}{l}\text { RMSEA } \\
\text { Cronbach }\end{array}$ \\
\hline
\end{tabular}

$(* * \mathrm{p}<0.001)$

Fuente: Basado en Ahire, Golhar y Waller (1996).

Nota: En la tabla se muestra las cargas factoriales en $1^{\circ}$ orden del constructo planeación estratégica. En este sentido las cargas factoriales de $1^{\circ}$ orden la variable de la latente oscila de entre $0.658_{(\lambda \mathrm{PE} 7)}$ a $0.821_{(\lambda \mathrm{PE} 2)}$, Todas las cargas factoriales del constructo correspondiente a la planeación estratégica son positivas y significativas. De la misma forma, la consistencia interna del instrumento medida a través del Alfa de Cronbach $(\alpha=0.89)$ resultó ser satisfactoria. En cuanto a los índices de bondad de ajuste del modelo $\left(X^{2}=41.23 ; g l=3\right.$; CFI=0.982; TLI=0.972; RMSEA=0.07) resultaron ser aceptables (Asparouhov, Hamaker \& Muthen, 2018; Bollen, 1989; Jöreskog \& Sörbom, 1981; Muthén \& Muthén, 1998-2007; Rigdon, 1996).

Tabla 3

Cargas factoriales estandarizadas y alfa de Cronbach del constructo creación de valor.

\begin{tabular}{ll}
\hline Ítems & Carga Factorial \\
\hline $\begin{array}{l}\text { CV1.- La empresa se preocupa en personalizar el producto de acuerdo con las necesida- } \\
\text { des de los clientes. }\end{array}$ & $0.754^{* *}$ \\
CV2.- En la empresa se emplea la innovación continua de productos / servicios. & $0.802^{* *}$ \\
CV3.- La prioridad de la organización está enfocada en la reducción de costos. & $0.656^{* *}$ \\
CV4.- El enfoque de la organización está enfocada en la calidad del producto / servicio. & $0.841^{* *}$
\end{tabular}


CV5.- Las prioridades de la empresa está en la investigación y desarrollo de nuevos productos / servicios.

\begin{tabular}{llll} 
CFI & TLI & RMSEA & $\square$ Cronbach \\
0.996 & 0.991 & 0.04 & 0.881 \\
\hline
\end{tabular}

$(* * \mathrm{p}<0.001)$

Fuente: Diseño Propio.

Nota: En la tabla se muestra las cargas factoriales en $1^{\circ}$ orden del constructo creación de valor. En este sentido las cargas factoriales de $1^{\circ}$ orden la variable de la latente oscila de entre $0.656_{(\lambda \mathrm{CV} 3)}$ a $0.841_{(\lambda \mathrm{CV} 4)}$, Todas las cargas factoriales del constructo correspondiente a la creación de valor son positivas y significativas. De la misma forma, la consistencia interna del instrumento medida a través del Alfa de Cronbach $(\alpha=0.881)$ resultó ser satisfactoria. En cuanto a los índices de bondad de ajuste del modelo $\left(X^{2}=7.80 ; g l=4\right.$; CFI=0.996; TLI=0.991; RMSEA=0.04) resultaron ser aceptables (Asparouhov, Hamaker \& Muthen, 2018; Bollen, 1989; Jöreskog \& Sörbom, 1981; Muthén \& Muthén, 1998-2007; Rigdon, 1996).

\section{Resultados}

Para evaluar el modelo de ecuación estructural (SEM) hipotético puesto en cuestión, se consideraron los siguientes índices de bondad y ajuste: Chi-cuadrado $\left(X^{2}=146.10 \mathrm{gl}=52\right)$, por lo que la prueba de Chi-cuadrada $\left(\mathrm{X}^{2} / g l=2.8 ; p<0.001\right)$ resultó ser satisfactoria, el Índice de Ajuste Comparativo (CFI $=0.969$ y TLI=0.960) y finalmente, la Aproximación de la Raíz Cuadrada del Error Cuadrático Medio (RMSEA= .06) por lo que el modelo resultó ser absolutamente deseable y aceptable (Asparouhov, Hamaker \& Muthen, 2018; Bollen, 1989; Jöreskog \& Sörbom, 1981; Muthén \& Muthén, 1998-2007; Rigdon, 1996), ver Figura 2.

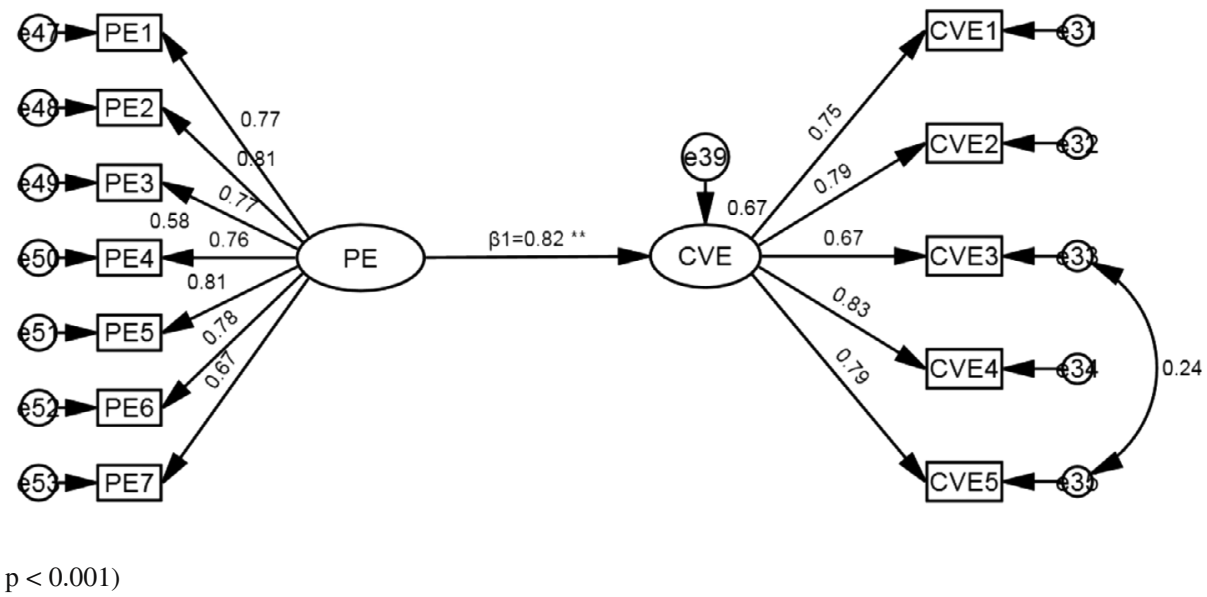

Figura 2. Cargas estructurales del modelo SEM hipotético puesto en cuestión. Diseño Propio. 
Nota: En la figura se muestra la carga estructural estandarizada de la variable exógena sobre las endógena y en base a la carga $\left(\beta_{1}\right)$ se analiza para evaluar la hipótesis establecida en la investigación. En este sentido, la carga estructural es positiva y significativa $\left(\beta_{1}=0.82 ; p<0.001\right)$. En cuanto a los índices de bondad y ajuste del modelo $\left(X^{2}=146.10 \mathrm{gl}=52\right.$; $\mathrm{CFI}=0.969$ y TLI=0.960; RMSEA=.06) resultaron absolutamente deseables (Asparouhov, Hamaker \& Muthen, 2018; Bollen, 1989; Jöreskog \& Sörbom, 1981; Muthén \& Muthén, 1998-2007; Rigdon, 1996). $\mathrm{PE}=$ Planeación Estratégica, $\mathrm{CVE}=$ Creación de Valor Empresarial.

A través de los resultados obtenidos del modelo SEM (ver figura 1) se demuestra que la planeación estratégica es un factor que influye positiva y significativamente sobre la creación de valor $\left(\beta_{1}=0.82 ; p<0.001\right)$. En este sentido, se cuenta con suficiente evidencia estadística para afirmar que la planeación estratégica es un factor influyente en la creación de valor en las microempresas de la región del bajío del estado de Guanajuato, México. Por lo tanto, no se rechaza la hipótesis $\mathrm{H}_{1}$.

De acuerdo con los resultados obtenidos por medio del análisis estadístico realizado a través de la correlación entre las variables latentes estudiadas, se demuestra que existe una correlación positiva y significativa $(r=0.686 ; p<0.001)$ entre las variables de la planeación estratégica y la creación de valor las empresas medida a través del coeficiente de correlación de Pearson (Bonett y Wright, 2000; Pearson, 1929, 1931). Por lo tanto, existe suficiente evidencia estadística para afirmar que la planeación estratégica y la creación de valor empresarial están fuertemente relacionadas entre sí. En este sentido, no se rechaza la hipótesis $\mathrm{H}_{2}$, ver tabla 1 .

\section{Conclusiones}

Actualmente el sector empresarial enfrenta a diversos cambios enmarcados por la tecnología, reformas políticas, económicas y aspectos sociales, estos últimos representan uno de los factores con mayor relevancia en la generación de cambios empresariales como organizacionales ante nuevas generaciones conocidas como Millenians (Hershatter \& Epstein, 2010).

Es menester promover en los nuevos emprendedores y empresarios de nuevas y anteriores generaciones, cursos y capacitaciones enmarcadas en la administración, innovación, creación de negocios, gestión empresarial como también de planeación estratégica que les permitan crear valor (Kachaner, King, \& Stewart, 2016) tanto en los productos y servicios que ofrecen a través de los negocios que tienen posicionados. De igual manera, se debe de fortalecer en las empresas que están en desarrollo (Aldianto, Anggadwita \& Umbara, 2018), con la finalidad de contar con herramientas administrativas y de gestión que les permitan posicionarse y mantenerse en el mercado y con ello evitar y minimizar el riesgo del fracaso de la microempresa, considerando la creación de valor como uno de los principales factores 
de diferenciación. De esta manera, se podrá hacer una diferencia en la competencia que se encuentra posicionada en el mercado (Åslund \& Bäckström, 2015).

La planeación estratégica es una herramienta que permite lograr los objetivos de calidad, dado que estos constituyen uno de los principales enfoques que persigue la empresa al planear y con ello, mejorar el desempeño empresarial (Kohtamäki et al., 2012). En este sentido, la planeación representa una guía para la toma de decisiones en los diferentes niveles de la organización, como también, representa una herramienta sustancial en las decisiones para el desarrollo de un modelo de negocio (Høgevold, Svensson, \& Padin, 2015). Por lo que, la planeación estratégica contribuye a tener claridad en el funcionamiento de la empresa, la manera en que genera ingresos, la identificación de los beneficios que proporciona a sus clientes, mediante las actividades, recursos y canales. Estos hallazgos son significativos ya que contribuyen con los hallazgos de las investigaciones realizadas por Høgevold, Svensson, y Padin, (2015) y Kohtamäki et al. (2012)

La planeación estratégica permite modelar el futuro de la empresa mediante la construcción de las directrices organizativas (Ljungberg \& Larsson, 2012) contenidas en la filosofía empresarial y en la matriz estratégica, seguidos por los gerentes y los empleados. En este sentido, es de vital importancia tener pleno conocimiento de la empresa y el entorno para tomar decisiones adecuadas y asertivas que conlleve a marcar prioridades en la innovación y en la mejora de la calidad (Jaafreh \& Al-abedallat, 2013; Ooi, 2014) del producto y servicio en el corto, mediano y largo plazo.

Asimismo, la planeación estratégica empresarial contribuye a la mejora continua de diversos indicadores claves (KPIs) como la reducción de los costos de producción, el sistema de calidad seguido, la investigación y el desarrollo en nuevas materias primas y nuevos productos y servicios, y la atención a las quejas de los clientes, siendo estos últimos pieza clave dentro de los grupos de interés. Además, es una herramienta que apoya de los dueños, trabajadores, proveedores y la comunidad donde se desempeña la empresa y se acuña su ventaja competitiva sostenible y sustentable (Ooi, 2014). Lo anterior es especialmente importante en las pequeñas empresas que van empezando.

Uno de los resultados obtenidos radica en la importancia de la gestión empresarial a través de la implementación de la planeación estratégica como uno de los factores influyentes en la creación de valor. De acuerdo con Åslund y Bäckström (2017) la gestión y la administración de nuevas firmas emprendedoras es impulsada por la necesidad, la oportunidad, el interés y la demanda al crear valor para el cliente. Por lo que la planeación estratégica tiene un papel fundamental en la creación de valor y ambos constructos influyen en el éxito de la firma emprendedora (Brozovic, Nordin \& Kindström, 2016). En este sentido, el uso e implementación 
de la planeación estratégica influye positiva y significativamente en la creación de valor de las microempresas emprendedoras.

Otro de los hallazgos encontrados en la presente investigación, radica principalmente en factores de calidad en la que se debe planear al innovar o desarrollar un producto o servicio (Leavy, 2018), es por ello que, al desarrollar las estrategias, así como las tácticas en la planeación deben estar enfocadas en el análisis financiero así como el FODA (Ghezzi, 2014) deben estar estrechamente vinculadas con valores de calidad (Åslund \& Bäckström, 2015) y con ello contribuir en el proceso de mejora continua en el proceso de planear estratégicamente para generar valor a través del emprendimiento (Aldianto, Anggadwita \& Umbara, 2018).

Adicionalmente otra aportación del estudio radica en la prioridad en que la empresa promueve la investigación y el desarrollo de nuevos productos / servicios enfocados puntualmente en su personalización como en la reducción de costos, de igual manera en la innovación continua de los mismos, pero también en garantizar la calidad Åslund y Bäckström (2017). Es decir, la generación y desarrollo de una planeación estratégica (Kohtamäki et al., 2012) que promueva y garantice la calidad en el producto o servicio que se promoverá a través del emprendimiento (Kachaner, King, \& Stewart, 2016) de las microempresas ayudará a su sustentabilidad.

Las decisiones empresariales no solo construirán las futuras fortalezas, sino que a su vez generarán flexibilidad al desarrollo de nuevas estrategias que contribuyan al crecimiento y desarrollo económico de la organización. Además, toda planeación estratégica (Ghezzi, 2014) debe contemplar los puntos débiles de los competidores y sus posibles respuestas, para aprovecharlas en la mejora de la participación del mercado (Romaniuk, Dawes \& Nenycz-Thiel, 2018). También hay que considerar como factores críticos de la planeación estratégica los sistemas de información, los procesos de control, la estructura organizativa, la cultura empresarial, etc. De no lograrse una buena implementación de la planeación estratégica del ámbito de las buenas intenciones a la acción, se corre el riesgo de que se convierta en un frustrante ejercicio directivo sin demasiados efectos prácticos. Especialmente en el caso de la generación de los Millenials estas herramientas son de utilidad para poder lograr que la organización que emprenden sea sustentable a largo plazo.

Cabe señalar que la creación de valor se le atribuye a la forma en que una empresa capitaliza y se beneficia a través del lucro. Sin embargo, una forma de lucrar y capitalizar es promover un valor agregado o crear valor a los productos (Hou \& Johri, 2018) o servicios a través de los intangibles (Hu, Ke, Guo \& Wen, 2015). En este sentido, la creación de valor está enfocada a través de promover y considerar las necesidades de los clientes, emplear la innovación, calidad, investigación y desarrollo de nuevos productos o servicios, así como 
emplear estrategias para reducir costos (Kirkbesoglu \& Ozder, 2015; Noordin et al., 2015; López \& Olivella, 2018) por lo que representa una ventaja competitiva para la microempresa.

La presente investigación es relevante e innovadora pues el tema ha sido poco estudiado, generando así nuevas líneas de conocimiento que deben ser exploradas, especialmente en el contexto de nuestro país. Adicionalmente, se busca sensibilizar y concientizar la importancia que tiene el desarrollar y aplicar estrategias adecuadas de administración y gestión empresarial a empresarios y emprendedores de microempresas, con la finalidad de desarrollar en ellos conocimientos y habilidades que contribuyan en la creación de valor. De esta manera, generar una sinergia que busque la mejora continua en los productos y servicios que ofrecen a través de la microempresa y logre se logre ser competitivo en el mercado.

Las limitaciones que se presentaron al realizar esta investigación radican en la participación de los jóvenes emprendedores de microempresas en el estado de Guanajuato. Si bien el Estado de Guanajuato es uno de los estados en México que se caracteriza por el emprendimiento de microempresas que promueven el desarrollo y crecimiento económico tanto de grandes o pequeños municipios del estado, lo que refuerza la relevancia del estudio, es necesario enfatizar la prudencia en la comprensión de los resultados de una manera particular a una realidad equivalente en los grandes centros económicos. La reivindicación intelectual para estudios futuros es ampliar la población estudiada durante períodos de tiempo para la comparación y evaluación de las barreras, prácticas, métodos y herramientas de gestión de conocimiento en las microempresas.

En esta investigación únicamente se valoraron dos variables que intervienen en el proceso empresarial. Es importante considerar que existen más variables intervinientes para la creación de valor en las empresas (el emprendedor, las variables micro y macroeconómicas, la existencia de un ecosistema emprendedor, etc.). Debido a que el tema es muy amplio, deberán seguir trabajos posteriores que amplíen el presente estudio para influir en las variables e mejora de las pequeñas que van naciendo y así lograr que puedan llegar a niveles de sustentabilidad y crecimiento.

\section{Agradecimientos}

Se hace un agradecimiento a la Universidad de Guanajuato y al Instituto Tecnológico de Celaya, al Departamento de Apoyo a la Investigación y al Posgrado de la Universidad de Guanajuato como también al Programa para el Desarrollo Profesional Docente, para el Tipo Superior (PRODEP), por brindarnos los recursos económicos y materiales, así como las facilidades para que el presente artículo fuera posible. 


\section{Referencias}

Ahire, S., Golhar, D. \& Waller M. (1996). Developmentand validation of TQM implementation constructs. Decision Sciences. 27(1). Pp.23-56. Doi: 10.1111/j.1540-5915.1996.tb00842.x

Aldianto, L., Anggadwita, G. \& Umbara, A. (2018). Entrepreneurship education program as value creation: Empirical findings of universities in Bandung, Indonesia. Journal of Science and Technology Policy Management. Doi: 10.1108/JSTPM-03-2018-0024

Ansoff, H. \& McDonnell, E. (1990). Implementing Strategic Management. Prentice-Hall. New York. NY.

Arend, R.J., Zhao, Y.L., Song, M., Im, S., 2017. Strategic planning as a complex and enabling managerial tool. Strategic Management Journal, 38, 1741-1752. Doi: 10.1002/smj.2420

Åslund, A. \& Bäckström, I. (2015). Creation of value to society - a process map of the societal entrepreneurship area, Total Quality Management \& Business Excellence. 26:3-4. pp. 385-399. Doi: 10.1080/14783363.2013.835897.

Åslund, A. \& Bäckström, I. (2017). Management processes and management's role in customer value creation. International Journal of Quality and Service Sciences. 9(2). Doi: 10.1108/IJQSS-11-2015-0074.

Asparouhov, T., Hamaker, E. \& Muthén, B. (2018) Dynamic Structural Equation Models. Structural Equation Modeling: A Multidisciplinary Journal. 25:3. Pp.359-388. Doi: 10.1080/10705511.2017.1406803

Bollen, K. (1989). Structural equations with latent variables. New York, NYS: John Wiley y Sons.

Bollen, K. \& Stine, R. (1992). Bootstrapping Goodness-of-Fit Measures in Structural Equation Models. Sociological Methods \& Research. 21. Pp. 205-229. Doi: 10.1177/0049124192021002004.

Bonett D. \& Wright T. (2000). Simple size requirements for estimating Pearson, Kendall and Sperman correlations. Psichometrika. 65(1). Pp. 23-28. Doi: 10.1007/BF02294183.

Brozovic, D., Nordin, F. \& Kindström, D. (2016). Service flexibility: conceptualizing value creation in service. Journal of Service Theory and Practice. 26 (6), pp.868-888, Doi: 10.1108/JSTP-09-2014-0219

Coelho, F. \& Augusto, M. (2010). Job characteristics and the creativity of frontline service employees. Journal of Service Research. 13 (4). pp. 426-438. Doi: 10.1177/1094670510369379

Cousens, A., Szwejczewski, M. \& Sweeney, M. (2009). A process for managing manufacturing flexibility. International Journal of Operations \& Production Management. 29 (4). pp. 357-385. Doi: 10.1108/01443570910945828

Cronbach, L. (1951). Coefficient alpha and internal structure of test. Psychometrica. 16. Pp. 297-335. Doi: 10.1007/ BF02310555.

Cronbach, L. (1984). Essentials of psychological testing (1a-5a edición). New York: Harper.

Davison, A., \& Hinkley, D. (1997). Bootstrap Methods and Their Application. Cambridge University Press: Cambridge.

Deming, W. (1986). Out of the Crisis. MIT Press: Cambridge, MA.

De la Garza, M., Zavala, M., \& López-Lemus, J. (2017). Competencias del emprendedor y su impacto en el desempeño organizacional. Universidad \& Empresa. 19(33). Pp. 53-74. Doi:10.12804/revistas.urosario.edu.co/ empresa/a.4811

De Toni, A. \& Tonchia, S. (1998). Manufacturing flexibility: a literature review. International Journal of Production Research. 36 (6). pp. 1587-1617. Doi: 10.1080/002075498193183

DOF (2009). Diario Oficial de la Federación: Acuerdo por el cual se establece la estratificación de la micro, pequeñas y medianas empresas. Emitido el 30 de Junio de 2009. Disponible en: https://dof.gob.mx/nota_detalle.php?codigo $=5551413 \&$ fecha $=28 / 02 / 2019 \&$ print $=$ true. Consultado el 15/11/2018.

Dries, N., Pepermans, R., \& De Kerpel, E. (2008). Exploring four generations beliefs about career: Is "satisfied" the new "successful"? . Journal of managerial Psychology, 8:23, 907-928. Doi: 10.1108/02683940810904394

Efron, B., \& Tibshirani, R. (1993). An Introduction to the Bootstrap. Chapman Hall: New York.

Frunzaru, V. \& Cismaru, D. (2018). The impact of individual entrepreneurial orientation and education on generation Z's intention towards entrepreneurship. Kybernetes. Doi: 10.1108/K-05-2018-0272

Ghezzi, A. (2014). The dark side of business models: the risks of strategizing through business models alone. Strategic Direction. 30(6). pp. 1 - 4. Doi: 10.1108/SD-03-2014-003 
Gónzalez, R. (2011). La incorporación de la Generación Y al mercado laboral: El caso de una Entidad Financiera de la ciudad de Resistencia. Palermo Business Review. 5. Pp. 67-93.

Gurau, C. (2012). A life-stage analysis of consumer loyalty profile: comparing Generation X and Millennial consumers. Journal Consumer Mark. 29 (2). Pp. 103-113. Doi: 10.1108/07363761211206357.

Gursoy, D., Geng-Qing, C. \& Karadag, E. (2013). Generational differences in work values and attitudes among frontline and service contact employees. International Journal of Hospitality Management. 32. Pp. 40-48. Doi: 10.1016/j.ijhm.2012.04.002.

Glaister, K. \& Falshaw, J. (1999). Strategic planning: still going strong. Long Range Planning. 32 (1). pp. 107-16. Doi: 10.1016/S0024-6301(98)00131-9.

Grönroos, C. \& Voima, P. (2013). Critical service logic: making sense of value creation and co-creation. Journal of the Academy of Marketing Science. 41 (2). pp. 133-50. Doi: 10.1007/s11747-012-0308-3

Hair, J., Hult, G., Ringle, C., \& Sarstedt, M. (2017). A Primer on Partial Least Squares Structural Equation Modeling (PLS-SEM). 2nd Ed., Sage: Thousand Oaks.

Hernández-Sampieri, R. \& Mendoza, C. (2018). Metodología de la investigación. Las rutas cuantitativa, cualitativa y mixta, Ciudad de México, México: Editorial Mc Graw Hill Education.

Hershatter, A., \& Epstein, M. (2010). Millennials and the world of work: An organization and management perspective. Journal of Business and Psychology. 25(2). pp.211-223. Doi: 10.1007/s10869-010-9160-y.

Høgevold, N., Svensson, G. \& Padin, C. (2015). A sustainable business model in services: an assessment and validation. International Journal of Quality and Service Sciences. 7(1). pp.17-33. Doi: 10.1108/IJQSS-09-2013-0037.

Hou, K. \& Johri, A. (2018). Intangible capital, the labor wedge and the volatility of corporate profits. Review of Economic Dynamics. 29. Pp. 216-234. Doi: 10.1016/j.red.2018.01.002.

Hu, Y., Ke, J., Guo, Z. \& Wen, J. (2015). Relationship between Intangible Capital, Knowledge and Maintenance Performance in a PSS Network: An Empirical Investigation. Procedia CIRP. Pp. 378 - 383. Doi: 10.1016/j. procir.2015.02.079.

Jaafreh, A. \& Al-abedallat, A. (2013). The effect of quality management practices on organizational perfomance in Jordan: An empirical study. International Journal of Financial Research. 4(1). Pp93-109. Doi: 10.5430/ijfr. v4n1p93.

Jöreskog Karl, y Sörbom Dag. (1981). LISREL: Analysis of linear structural relationships by maximum likelihood and least squares methods. Chicago, IL: National Educational Resources.

Kachaner, N., King, K. \& Stewart, S. (2016). Four best practices for strategic planning. Strategy \& Leadership. 44 (4). pp. 26 - 31. Doi: 10.1108/SL-06-2016-0046.

Kahla, F. (2017). Implementation of a balanced scorecard for hybrid business models an application for citizen renewable energy companies in Germany. International Journal of Energy Sector Management, Vol. 11 Issue: 3, pp.426-443, Doi: 10.1108/IJESM-09-2016-0004.

Kaplan, S. \& Beinhocker, E. (2003). The real value of strategic planning. MIT Sloan Management Review. 44 (2). pp. 71-76.

Khan, G. \& Al-Buraki, E. (1992). Strategic planning in Bahrain. Management Decision, 30 (6): pp. 3-9. Doi: $10.1108 / 00251749210015599$.

Kim, S. (2018). Managing millennials' personal use of technology at work. Business Horizons, 61(2), 261-270. Doi: 10.1016/j.bushor.2017.11.007.

Kirkbesoglu, E. \& Ozder, E. (2015). The Effects of Organizational Performance on the Relationship between Perceived Organizational Support and Career Satisfaction: An Application on Insurance Industry. Journal of Management Research. 7(3). Pp. 35-50. Doi: 10.5296/jmr.v7i3.7094.

Kohtamäki, M., Kraus, S., Mäkelä, M. \& Rönkkö, M. (2012). The role of personnel commitment to strategy implementation and organisational learning within the relationship between strategic planning and company performance. International Journal of Entrepreneurial Behavior \& Research, 18(2). pp.159-178. Doi: $10.1108 / 13552551211204201$ 


\section{J. A. López Lemus y M. T. De la Garza Carranza / Contaduría y Administración 65(3), 2020, 1-23}

http://dx.doi.org/10.22201/fca.24488410e.2020.2312

Krumwiede, K. \& Charles, S. (2006). Finding the right mix: How to match strategy and management practices to enhance firm performance. Strategic Finance. 87(10) pp. 37.

Kupperschmidt, B.R. (2000). Multigeneration Employees: Strategies for Effective Management, Health Care Manager, 19(1) pp. 65-76. Doi: 10.1097/00126450-200019010-00011

Lee, V., Ooi, K. Sohal, A. \& Chong, A. (2012). Structural relationship between TQM practices and learning organisation in Malaysia's manufacturing industry. Production Planning \& control: The Management of Operations. 2310-11. Pp.885-902. Doi: 10.1080/09537287.2011.642209.

Leavy, B. (2018). Cost innovation - a value-creation strategy to transform over-priced industries. Strategy \& Leadership. 46 (6). pp.3-13. Doi:10.1108/SL-09-2018-008

Lissitsa, S. \& Kol, O. (2016). Generation X vs. Generation Y - A decade of online shopping. Journal of Retailing and Consumer Services. 31. Pp. 304-312. Doi: 10.1016/j.jretconser.2016.04.015.

Ljungberg, A., \& Larsson, E. (2012). Processbaserad verksamhetsutveckling : varför, vad, hur? (in Swedish). Lund: Studentlitteratur AB.

López, J. (10 de 10 de 2018). El Financiero: Fracasan en México 75\% de emprendimientos. Disponible en http:// www.elfinanciero.com.mx: http://www.elfinanciero.com.mx/empresas/fracasan-en-mexico-75-de-emprendimientos. Consultado: 15/11/2018

López-de-Alba, P., Zavala, M., De la Garza, M., López-Lemus, J., \& Ramos, C. (2016). Causas de fracaso en empresas sociales mexicanas. The Failure Institute. pp. 1-29.

López-Lemus, J. (2019). Political ability and strategies of integrative and distributive negotiation as influential factors in entrepreneurial self-efficacy in Mexico. Cuadernos de Gestión. 19(2). pp. 113-136. Doi: 10.5295/ cdg.180943j1

López-Lemus, J. \& De la Garza, M. (2019). The practices of business management, innovation and entrepreneurship: influencing factors in the performance of entrepreneur firms. NovaScientia. No. 22. Vol. 11(1). Pp. 357383. Doi: 10.21640/ns.v11i22.1795.

López, J. \& Olivella, V. (2018). The importance of intangible capital for the transmission of financial shocks. Review of Economic Dynamics. Doi: 10.1016/j.red.2018.04.004.

Messick, S. (1980). Test validity and ethics of assessment. American Psychologist. 35. Pp. 1012-1027. Doi: 10.1037//0003-066X.35.11.1012.

Muthén, L. y Muthén, B. (1998-2007). Mplus Version 5.0 statistical analysis with latent variables: User's Guide (Fourth ed.). Los Angeles, CA: Muthen y Muthen.

Muthén, B. (2001). Second-Generation structural equation modeling with a combination of categorical latent variables: New opportunities for latent class/latent growth modeling. In L. M. Collins y A. Sayer (Eds.), New Methods for the Analysis of Change (pp. 289-332). Washington, D.C.

Muthén, B. (2002). Beyond SEM: General latent variable modeling. Behaviometrika, 29(1), 81-117. Doi: 10.2333/ bhmk.29.81

Nitya, R. \& Anand S. (2016). A study on generational differences in work values and person- organization fit and its effect on turnover intention of Generation Y in India. Management Research Review. 39 (12). pp.1695-1719. Doi: 10.1108/MRR-10-2015-0249

Noordin, R., Zainuddin, Y, Fuad, Mail, R. \& Kaziemah, N. (2015). Performance outcomes of strategic management accounting information usage in Malasia: Insights from electrical and electronics companies. Procedia Economics and Finance. 31. Pp. 13-25. Doi: 10.1016/s2212-5671(15)01302-2.

Oliva, F. \& Kotabe, M. (2019). Barriers, practices, methods and knowledge management tools in startups. Journal of Knowledge Management. Doi: 10.1108/JKM-06-2018-0361

Ooi,K. (2014). TQM: A facilitator to enhance knowledge management? A structural analysis. Expert Systems with Applications. 41 pp.5167-5179. Doi: 10.1016/j.eswa.2014.03.013.

Owen, R., Haddock-Millar, J., Sepulveda, L., Sanyal, C., Syrett, S., Kaye, N., Deakins, D. (2019) "The Role of Mentoring in Youth Entrepreneurship Finance: A Global Perspective 1 "In Creating Entrepreneurial Space: 
Talking Through Multi-Voices, Reflections on Emerging Debates. Published online: 30 May 2019; 115-135. Doi: 10.1108/S2040-72462019000009B007.

Parment, A. (2013). Generation Y vs. Baby Boomers: shopping behavior, buyer involvement and implications for retailing. Journal Retailing and Consumer Services. 20 (2). Pp.189-199. Doi: 10.1016/j.jretconser.2012.12.001.

Parnell, J. A., Lester, D. L., \& Menefee, M. L. (2000). Strategy as a response to organizational uncertainty: an alternative perspective on the strategy-performance relationship. Management Decision, 38(8), 520-530.Doi: $10.1108 / 00251740010352811$.

Pearson, E.S. (1929). Some notes on sampling tests with two variables. Biometrika, 21, 337-360. Doi: $10.2307 / 2332565$

Pearson, E.S. (1931). The test of significance for the correlation coefficient. Journal of the American Statistical Association, 26, 128-134. Doi: 10.2307/2278641

Peng, M. (2012). Global Strategy. New York.: Free Press.

Perrini, F. \& Vurro, C. (2010). Corporate Sustainability, Intangible Assets Accumulation and Competitive Advantage. Symphonya. Emerging Issues in Management. 2. pp. 25-38 Doi: 10.4468/2010.2.03perrini.vurro.

Pillai, D. \& Dam, L. (2019) From baby boomer to millennials: the changing flavor of entrepreneurial traits. Emerald Emerging Markets Case Studies, 9 (1). pp.1-19, Doi: 10.1108/EEMCS-07-2017-0186

Porter, M. \& Kramer, M. (2006). Strategy and Society: The Link Between Competitive Advantage and Corporate Social Responsibility. Harvard Business Review. 84 (12). pp. 78-92.

Posch, A. \& Garaus, C. (2019). Boon or Curse? A Contingent View on the Relationship between Strategic Planning and Organizational Ambidexterity. Long Range Planning. Pp. 1-56. Doi: 10.1016/j.lrp.2019.03.004.

Prajogo, I. \& McDermott, C. (2008). The relationships between operations strategies and operations activities in service context. International Journal of Service Industry Management. 19 (4). pp. 506-520. Doi: $10.1108 / 09564230810891932$

Prajogo, I. \& Sohal, S. (2003). The relationship between TQM practices, quality performance and innovation performance: an empirical examination. International Journal of Quality and Reliability Management. 20(8). Pp.901-918. Doi: 10.1108/02656710310493625.

Prior, J., Hubbard, P. \& Rai, Y. (2016). Using residents worries about technology as a way of resolving environmental remediation dilemmas. Science of total environment. Pp. 1-18. Doi: 10.1016/j.scitotenv.2016.12.035.

Rigdon, E. (1996). CFI versus RMSEA: A comparison of two fit indexes for structural equation modeling. Structural Equation Modeling: A Multidisciplinary Journal. 3(4), 369-379. Doi: 10.1080/10705519609540052.

Romaniuk, J., Dawes, J. \& Nenycz-Thiel, M. (2018). Modeling brand market share change in emerging markets. International Marketing Review. 35(5). pp.785-805. Doi:10.1108/IMR-01-2017-0006

Sánchez, R. \& Cerdán, A. (2002). Creación de valor empresarial a través del Capital Intelectual y la Gestión del Conocimiento. Revista Gestión. 21. Pp. 18-24.

Shammari, H. \& Hussein, R. (2008). Strategic planning in emergent market organizations: empirical investigation. International Journal of Commerce and Management. 18 (1). pp. 47-59. Doi: 10.1108/10569210810871489.

Spender, J., Corvello, V., Grimaldi, M. \& Rippa, P. (2017). Startups and open innovation: a review of the literature. European Journal of Innovation Management. 20(1), pp. 4-30. Doi: 10.1108/EJIM-12-2015-0131.

Teberga, P., Oliva, F. \& Kotabe, M. (2018). Risk analysis in introduction of new technologies by start-ups in the brazilian market. Management Decision. 56 (1). pp. 64-86. Doi: 10.1108/MD-04-2017-0337.

Teixeira, G. \& Canciglieri, O. (2018). Application of strategy planning method to integrated development sustainable product process (PEPDIPS). In: Giannetti, B.F., Almeida, C.M.V.B., Agostinho, F. (Eds.), Advances in Cleaner Production, 7th International Workshop, Barranquilla, pp. 28-38.

Teixeira, G. \& Canciglieri, O. (2019). How to make strategic planning for corporate sustainability? Journal of Cleaner Production. Pp. 1421-1431. Doi: 10.1016/j.jclepro.2019.05.063.

Vecchiato, R. (2019). Scenario planning, cognition, and strategic investment decisions in a turbulent environment. Long Range Planning. 52(5). Pp.1-17. Doi:10.1016/j.1rp.2019.01.002. 
Verdú-Jover, A., Lloréns-Montes, F. \& García-Morales, V. (2004). The concept of fit in services flexibility research: an empirical approach. International Journal of Service Industry Management. 15 (5). pp. 499-514. Doi: $10.1108 / 09564230410564957$

Viscarri, J. (2011). Modelo de creación de valor para el cliente. In Memoria del XVI Congreso Internacional de Contaduría, Administración e Informática (pp. 1-17). Disponible en: https://upcommons.upc.edu/bitstream/ handle/2117/16640/Viscarri_modelo_creacion_valor_cliente.pdf Consultado el 12/11/2018.

von Gelderen, M., Frese, M., \& Thurik, R. (2000). Strategies, uncertainty and performance of small business startups. Small Business Economics, 15(3), pp. 165-181. Doi: 10.1023/A:1008113613597.

Wheadon, M. Duval-Couetil, N. (2017). Entrepreneuring gender diversity in entrepreneurship through critical theory and reflexivity. International Journal of Gender and Entrepreneurship, Vol. 9 (2). pp.188-202. Doi: 10.1108/IJGE-02-2017-0010

Wolf, C., \& Floyd, S. (2017). Strategic planning research: Toward a theory-driven agenda. Journal of Management, 43, 1754-1788. Doi: 10.1177/0149206313478185.

Wolff, J. A., \& Pett, T. L. (2000). Internationalization of small firms: An examination of export competitive patterns, firm size, and export performance. Journal of small business management, 38(2), 34.

\section{Anexo}

Tabla 4

Cuestionario del constructo Planeación Estratégica

\section{Planeación Estratégica}

PE1.- Nuestra organización tiene una misión que se ha comunicado a través de la compañía y es apoyada por nuestros empleados.

PE2.- Nuestra organización tiene un proceso de planificación integral y estructurado que establece y regula regularmente las metas a corto y a largo plazo.

PE3.-Nuestra organización siempre incorpora las capacidades de los proveedores y las necesidades de otras partes interesadas, incluida la comunidad, cuando desarrollamos nuestros planes, políticas y objetivos.

PE4.- Nuestra organización tiene una declaración por escrito una estrategia que abarca las operaciones de negocio que este articulado y acordado por nuestro gerente superior.

PE5.- Los planes estratégicos (y los planes tácticos) están vinculados a los valores de calidad.

PE6.- Se hace inclusión de mejoras continuas de la calidad en el proceso de planificación.

PE7.- Las quejas de los clientes fueron analizadas y utilizadas para la mejora del producto / servicio que ofrece la organización.

Fuente: Basado en Ahire, Golhar y Waller (1996). 


\section{Tabla 5}

Cuestionario del constructo Creación de Valor

Creación de Valor

CV1.- La empresa se preocupa en personalizar el producto de acuerdo con las necesidades de los clientes.

CV2.- En la empresa se emplea la innovación continua de productos / servicios.

CV3.- El enfoque de la organización está enfocada en la reducción de costos.

CV4.- El enfoque de la organización está enfocada en la calidad del producto / servicio.

CV5.- Las prioridades de la empresa está en la investigación y desarrollo de nuevos productos / servicios.

Fuente: Basado en Noordin et al. (2015). 